\title{
VIII Jornadas internacionales de cultura de los cuidados: Violencia de género, mutilación genital femenina y cuidados de salud
}

VIII International conference on the culture of care: gender violence, female genital mutilation and health care

VIII Conferência internacional sobre a cultura do cuidado: violência de gênero, mutilação genital feminina e atenção à saúde

Alicante, 13 y 14 de Junio de 2019

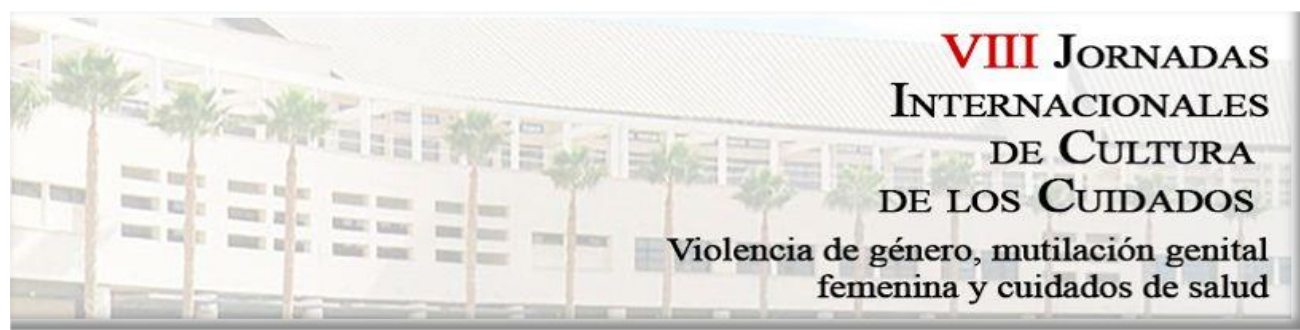

José Siles González

Catedrático de la Facultad de Ciencias de la Salud de la Universidad de Alicante

Cómo citar este documento de difusión en edición digital: Siles-González, J. (2018). VIII Jornadas Internacionales de Cultura de los Cuidados: Violencia de género, mutilación genital femenina y cuidados de salud (2019). Cultura de los Cuidados (Edición digital), 22(52). Recuperado de $<$ http://dx.doi.org/10.14198/cuid.2018.52.26>

Correspondencia: Departamento de enfermería. Universidad de Alicante. 03080-Alicante (España) Correo electrónico: jose.siles@ua.es

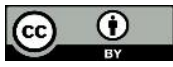

\section{ABSTRACT}

This document disseminates the activities of the next Congress of Culture of Care dedicated to research on gender violence, female genital mutilation and health care

Keywords: Gender violence, nursing, care, female genital mutilation.

\section{RESUMO}

Este documento divulga as atividades do próximo Congresso de Cultura de Cuidado dedicado à pesquisa sobre violência de gênero, mutilação genital feminina e assistência médica.

Palavras-chave: Violência de gênero, enfermagem, cuidado, mutilação genital feminina. 


\section{RESUMEN}

En este documento se difunden las actividades de las próximas jornadas de Cultura de los Cuidados dedicadas a la investigación sobre Violencia de género, mutilación genital femenina y cuidados de salud.

Palabras clave: Violencia de género, enfermería, cuidados, mutilación genital femenina.

\section{OBJETIVOS}

Con la celebración de este evento científico, nos hemos propuesto:

- Identificar los factores históricos, fenomenológicos, culturales y sociales que inciden en la violencia de género $y$ en la mutilación genital femenina y su impacto en las mujeres que la han padecido.

- Promover la reflexión y el debate entre profesionales de la salud y sociales sobre las diferentes concepciones de educación y cuidados de salud con el fin de afrontar la violencia de género y la mutilación genital femenina.

- Analizar el fenómeno de la violencia de género y la mutilación genital femenina desde una perspectiva histórica, fenomenológica, antropológica y social.

- Conocer las vivencias experimentadas por mujeres padecido violencia de género y la mutilación genital femenina.

- Establecer los factores que facilitan o dificultan las prácticas de violencia de género y/o mutilación genital femenina.

- Implicar a las mujeres que han sufrido violencia de género y/o mutilación genital femenina facilitando su participación y empoderamiento en la lucha activa contra dichas prácticas.

- Potenciar la comunicación transcultural entre mujeres que han padecido violencia de género y/o mutilación genital femenina.

- Contribuir al cambio de conocimientos, actitudes y habilidades de los profesionales de salud para facilitar el afrontamiento preventivo de la violencia de género y la mutilación genital femenina.

- Explorar los recursos políticos y sociales de los municipios, comunidades y país en la erradicación de la violencia de género y mutilación genital femenina.

\section{PROGRAMA}

Puede descargar el programa en formato PDF en el siguiente enlace: http://www.culturadeloscuidados.org/.

\section{JUEVES 13 JUNIO 2019}

\section{8:30h}

Entrega de documentación.

\section{9:00h}

Colocación de Póster.

Coordinadoras: Judith Gómez Carrillo y Zhenet Ilieva Stefanova

- Lugar: Hall Edificio de Enfermería. 
Acto de apertura.

$$
\text { 10:00h - 11:00h }
$$

\section{Conferencia Inaugural.}

- D. a Adriana Kaplan Marcusán. Coordinadora del GIPE/GTP - Grupo Interdisciplinar para la Prevención y el Estudio de las Prácticas Tradicionales Perjudiciales. Departamento de Antropología Social y Cultural UAB.

\section{1:00h}

Pausa - Café.

\section{1:30h - 12:30h}

Mesa Redonda N.o 1.- Fenomenología: Experiencias/vivencias sobre violencia de género y mutilación genital femenina.

- Modera: Judith Gómez Carrillo

- D. a Fátima Djarrá. Médicos del mundo de Navarra. Mi experiencia con la MGF. Estrategias para afrontarla y superar sus consecuencias.

- D.a Asha Ismail. Save Generation. Características de la Mutilación Genital Femenina. Mis vivencias.

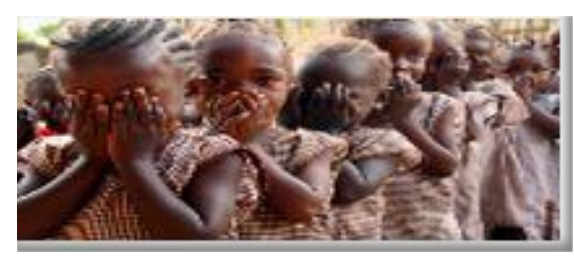

\section{2:30h}

Presentación de Comunicaciones.

- Modera: D.a Ana Lucia Noreña.

\section{4:00h}

\section{Almuerzo.}

\section{6:00h}

Mesa Redonda N.o 2.- Historia: Historia de la violencia de género y la mutilación genital femenina.

- Modera: D. Luis Cibanal Juan.

- Lugar: Aula A2/E02 Aulario II.

- Participan:

- Dr. José Siles González. Catedrático del departamento de enfermería. Universidad. Universidad de Alicante. Historia de la mutilación genital femenina.

- Dra. María Reig Alcaraz. Historia reciente de la mutilación genital femenina en España.

- Dr. Manuel Moreno Preciado. Salud, pobreza y exclusión social: otra mirada sobre la violencia de género". 


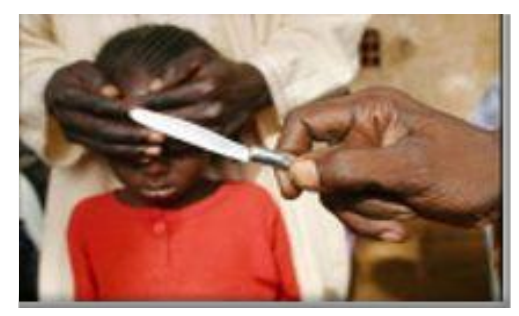

17:30h

\section{Presentación de Comunicaciones.}

- Modera: D. a Isabel Casabona Martínez.

\section{8:00h - 20:00h}

\section{Mesa debate:}

\section{Modera: Isabel Casabona Martínez}

- Nuria Gregori Flor. Enfermera y antropóloga. Departamento de docencia e investigación. Hospital General de Elche. El género en el cuerpo: versiones occidentales de la mutilación genital femenina.

- Aula A2/E02 Aulario II.

\section{Acto lúdico.}

\section{0:00h}

\section{VIERNES 14 JUNIO 2019}

$$
\text { 9:00h - 10:00h }
$$

Mesa redonda N.o 3.- Antropología de los Cuidados, violencia de género y mutilación genital femenina

- Modera: D. César Hueso Montoro.

- Lugar: Pendiente de confirmar.

- Participan:

- Dra. Modesta Salazar Agulló. Instituto Universitario de Investigación de Estudios de Género (IUIEG). Profesora colaboradora del Departamento de enfermería. Universidad de Alicante: Los úteros de alquiler. Otra violencia de género.

- Dra. Carmen Vives Cases: Determinantes sociales de la violencia de género.

- Montserrat Angulo Perea. Enfermera, matrona y vicepresidenta del Colegio de Enfermería de Alicante.: Mutilación genital femenina desde la perspectiva de una matrona.

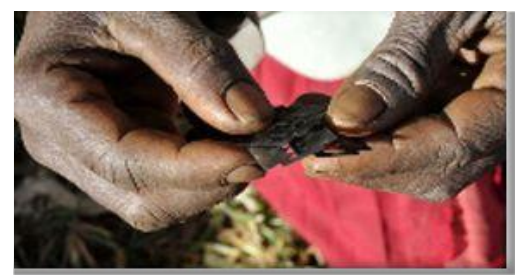




\section{$10: 45 h$}

Pausa Café.

\section{1:00h}

Defensa Posters.

Responsable: $\mathrm{J}$.

\section{$11: 15 h$}

Presentación de Comunicaciones.

Modera: Lola Gil.

\section{4:00h}

Almuerzo de trabajo.

\section{6:30h - 18:00h}

Mesa redonda N. ${ }^{\circ}$ 4.- Teoría y método en violencia de género y MGF Modera: Modesta Salazar Agulló

- D. Cadidjato Baldé. Graduada en enfermería e investigadora de la MGF en Guinea Bissau: Estudio de casos sobre la Mutilación Genital Femenina "Jadare" en la cultura fulbe (Guinea Bissau).

- Ismael Jiménez Ruiz: La mirada masculina en la MGF.

- Emilia Tonda Monllor. Catedrática de E. U. Facultad Educación: Universidad de Alicante: Educación, familia y violencia de género.

\section{8:00h- 20:00 h}

Presentación de Comunicaciones.

Modera: Miguel Ángel Fernández Molina

$$
\text { 18:00h - 20:00h }
$$

Presentación libros. Fallo Premio VIDA Y SALUD. Responsable. Aula A2/E02 Aulario II

\section{0:00h- 21:00h}

Conferencia de Clausura.

- D. a María Ballester Cerezo. Experiencias sobre violencia de género en el contexto de la Asociación "Elche Acoge". Psicóloga de la Asociación "Elche Acoge".

- Lugar: Salón de Actos del edificio Germán Bernácer.

\section{Preinscripción}

\section{Coste de inscripción: $175 €$}

Rellene el formulario para solicitar su preinscripción. Se contactará con usted con más detalles para completar su inscripción. http://www.culturadeloscuidados.org/inscripcion.html 
Inscripción a las VIII Jornadas Internacionales de Cultura de los Cuidados Lema: Violencia de Género, mutilación genital femenina y cuidados de salud. Fecha: 13 y 14 de junio de 2019.

Inscripción "on line": http://www.culturadeloscuidados.org/inscripcion.html.

Más información: jornadas@ @ulturadeloscuidados.org - 965123622.

Organizan:

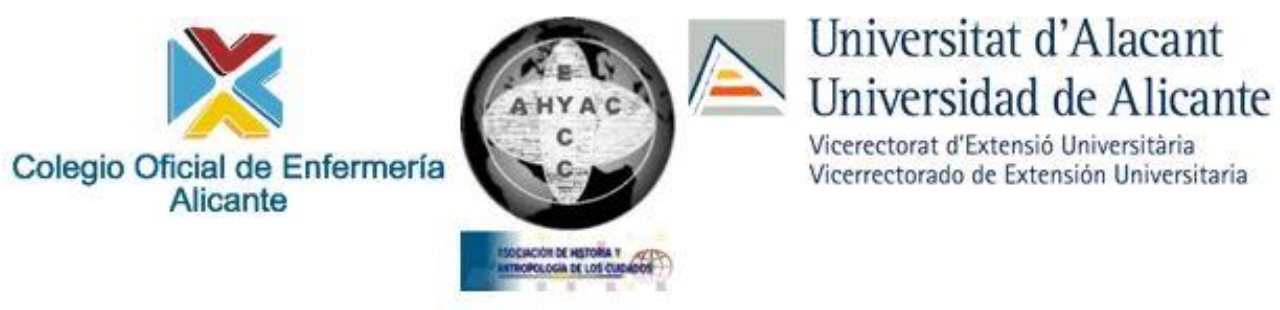

Colabora: 